\title{
Propuesta de un Instrumento de Autoevaluación del Desempeño Organizacional en Instituciones Públicas de Educación Superior en México
}

\author{
Felipe Machorro* y María V. Romero \\ Instituto Tecnológico Superior de Tierra Blanca, Prol. Av. Veracruz s/n, Tierra Blanca, Veracruz, México. \\ (e-mail: felipe.machorro@itstb.edu.mx; vromero@itstb.edu.mx)
}

* Autor a quien debe ser dirigida la correspondencia

Recibido Oct. 21, 2016; Aceptado Dic. 26, 2016; Versión final Ene. 18, 2017, Publicado Jun. 2017

\begin{abstract}
Resumen
El objetivo de este documento es presentar la propuesta de un instrumento de autoevaluación del desempeño organizacional aplicable a las instituciones públicas de educación superior en México. El cuestionario fue diseñado con base en las cuatro perspectivas del modelo de cuadro de mando integral de Kaplan y Norton, constó de 50 ítems y se aplicó a una muestra de 74 universidades en México. Los resultados indican una alta confiabilidad del instrumento en las cuatro dimensiones, una percepción de mejor desempeño en dos perspectivas: de aprendizaje y crecimiento y de clientes sobre las dos restantes (financiera y procesos internos). Se encontraron correlaciones significativas para todas las perspectivas.
\end{abstract}

Palabras clave: desempeño organizacional; universidad; cuestionario; cuadro de mando integral.

\section{Proposal of an Instrument for Self-Evaluation of Organizational Performance in Public Institutions of Higher Education in Mexico}

\begin{abstract}
The purpose of this document is to present a proposal of a self-assessment instrument of organizational performance applicable to Public Higher Education Institutions in Mexico. The questionnaire was designed based on four perspectives of Kaplan \& Norton's Balanced Scorecard model. It consisted of 50 items and was applied to a sample of 74 universities in Mexico. The results indicate a high reliability of the instrument in the four dimensions, a perception of better performance in learning and growth and client on financial and internal processes. Significant correlations for all perspectives were found.
\end{abstract}

Keywords: organizational performance; university; questionnaire; balanced scorecard. 


\section{INTRODUCCIÓN}

En el contexto de las instituciones públicas de educación superior (IPES), el desempeño organizacional cobra relevancia debido al papel fundamental que estas organizaciones tienen en la formación de capital humano que sustenta el desarrollo económico de las naciones (Wu, et al., 2012). Más aún, en las IPES ha aumentado el interés en la evaluación del desempeño como consecuencia del incremento de la competencia a raíz del crecimiento del número de instituciones públicas y privadas que origina mayor presión gubernamental y social para demostrar su rendimiento en función del costo que generan a la sociedad, exigiéndose resultados tangibles a corto y largo plazo (Sarrico et al., 2010).

Tradicionalmente, el apoyo gubernamental para la enseñanza e investigación se ha justificado sobre la base de que producen un bien público, donde los beneficios específicos están dispersos y los resultados no son inmediatos. Sin embargo, cada vez es mayor la tendencia de exigir resultados tangibles en el corto plazo (Guthrie y Neumann, 2007). Un resultado de esta presión es el intento en la educación superior de aplicar los conceptos empresariales en su gestión, sobre todo el enfoque a las necesidades del cliente (Mustafa y Dalen, 2006; Pounder, 1999), por lo que cada vez más se utilizan modelos de medición del desempeño creados en entornos empresariales tales como las certificaciones de calidad de la Organización Internacional para la Estandarización (International Organization for Standardization, ISO) (Tlapa, Limón y Báez, 2009), el cuadro de mando integral (Balanced Scorecard, BSC) (Shun-Hsing, Ching-Chow y Jiun-Yan, 2006), y el modelo de excelencia de la Fundación Europea de la Gestión de la Calidad (European Foundation for Quality Management, EFQM) (Tarí y De Juana Espinosa, 2007). Sin embargo, al igual que en las empresas, la elección de indicadores de desempeño en las instituciones de educación superior es importante pero compleja y en ocasiones controversial. La presencia de diferentes modelos de evaluación en las universidades, ha generado multiplicidad de indicadores para tal propósito, sin que exista un consenso sobre los factores clave de medición (Palomares-Montero y García-Aracil, 2011). Los primeros trabajos que intentaron encontrar indicadores adecuados de desempeño para medir los resultados en las instituciones de educación superior fueron hechos por Mertens (1979), en el cual se proponen tres categorías de indicadores de desempeño: en relación a los gastos (por ejemplo, costo por estudiante), capacidad (espacio disponible) y rendimiento estudiantil (semestres promedio de realización de estudios). Por su parte, Jarrat (1985) sugiere tres categorías: desempeño interno, desempeño externo y desempeño operativo.

En general, se identifica que el criterio más frecuente para medir el desempeño de las universidades es la enseñanza y el aprendizaje, relacionados con el objetivo primordial de proveer educación de calidad a sus usuarios y formar recursos humanos calificados para el sector productivo (Capaldi y Abbey, 2011; lqbal et al., 2011). De igual manera, la investigación, vista como la capacidad de las universidades para generar nuevos conocimientos, se ha propuesto como indicador para evaluar su desempeño mediante rankings que clasifican universidades en función de su producción científica (Capaldi et al., 2010; Shin, 2009); bajo este enfoque, Aghion et al., (2010) analizan la influencia de la autonomía universitaria y el ambiente de competencia que enfrentan las instituciones en su desempeño en investigación. Aunado a lo anterior, y de acuerdo con el contexto actual de competencia y exigencia social, las universidades deben tener viabilidad financiera (Guthrie y Neumann, 2007; Xing, 2009) y sustentabilidad ambiental (Baboulet y Lenzen, 2010). De esta forma, se puede apreciar que el desempeño organizacional en las universidades resulta un concepto multidimensional y de difícil medición.

Bajo esta perspectiva, la situación de las IPES mexicanas no es distinta a la del resto del mundo: las universidades mexicanas se han incorporado a los esquemas empresariales mediante el establecimiento de sistemas de gestión de la calidad, con la finalidad de certificar sus procesos. No obstante, estas certificaciones y acreditaciones externas atienden al concepto extrínseco de la calidad (Sarrico et al., 2010), con lo que queda pendiente la evaluación del desempeño en función de la satisfacción de sus estudiantes y de los intereses tanto de los actores directos como de la sociedad en general, por lo que crece la importancia de aplicar mecanismos para asegurar el mejoramiento del desempeño de las instituciones educativas de manera más integral. En ese sentido, existen algunos instrumentos diseñados para la autoevaluación del desempeño organizacional en la educación superior, tales como el cuestionario de la EFQM o la adaptación del modelo de Gratton en Chile (Barra y Gómez, 2013). Sin embargo, aún es necesario un mayor desarrollo de la investigación al respecto, y, en el caso de México, el diseño de un instrumento acorde a las características de las instituciones del país. Por lo anterior, el objetivo del presente documento es presentar una propuesta de un instrumento para la autoevaluación del desempeño organizacional en las instituciones públicas de educación superior en México, el cual fue desarrollado con base en el modelo de Cuadro de Mando Integral de Kaplan y Norton. 


\section{CUADRO DE MANDO INTEGRAL Y DESEMPEÑO ORGANIZACIONAL}

El cuadro de mando integral (CMI), es uno de los sistemas más utilizados como instrumento en control de gestión para las empresas de todo el mundo. Fue propuesto por Kaplan y Norton (1992), quienes plantean cuatro perspectivas en las cuales desarrollar los objetivos e indicadores: la perspectiva financiera contempla examinar los resultados económicos buscando maximizar los beneficios, relacionados en mayor medida con la información contable de la organización; la perspectiva del cliente considera la imagen, calidad, relaciones, precio o aquellos factores que son importantes para mantener la satisfacción de éste; la perspectiva de procesos internos representa la cadena de procesos desarrollados para la producción de un bien o servicio; por último, la perspectiva de aprendizaje y crecimiento, desarrolla indicadores para impulsar la inversión en nuevas tecnologías, mejoras o perfeccionamiento en los resultados de las tres perspectivas anteriores.

Este modelo, nacido en el ámbito empresarial, ha sido adaptado en distintos contextos, por ejemplo, en el de las universidades. Tal es el caso del trabajo de Xing (2009), quien propone el uso del CMI en la medición del desempeño organizacional de siete universidades en China. Para tal efecto en la perspectiva financiera considera como indicador la proporción entre el gasto y el ingreso; en la perspectiva clientes incluye la satisfacción social, de los empleadores, de padres y estudiantes; los procesos internos evaluados fueron el desempeño de la docencia y la investigación científica; finalmente, en la perspectiva de aprendizaje y crecimiento se evaluó la satisfacción de la planta docente.

Otros ejemplos del diseño y aplicación de CMI en universidades se identifican en diversos países; en Inglaterra Philbin (2011) adaptó las cuatro perspectivas: por principio, la financiera que consideró los fondos por programa; posteriormente la perspectiva de las partes interesadas (stakeholders); la tercer perspectiva mide las capacidades de la institución (personal adscrito y equipo); y la perspectiva de aprendizaje y crecimiento, la cual considera los productos de investigación (artículos científicos y conferencias). Por su parte, Hladchenko (2015) presenta un análisis comparativo del diseño de Cuadros de Mando Integral en tres universidades de Alemania y una en Austria. Sus conclusiones al respecto fueron que el CMI provee una visión sistemática de la estrategia de la institución, al tiempo que la traduce en un conjunto de indicadores de para la medición del desempeño organizacional. Finalmente, en Egipto Mohamad (2016) evaluó el desempeño de ocho facultades acreditadas de la universidad Assiut. Las dimensiones utilizadas para tal estudio fueron cinco: perspectiva financiera, perspectiva de los clientes y beneficiarios, perspectiva de procesos internos, perspectiva de aprendizaje y crecimiento, y perspectiva de desempeño social.

\section{METODOLOGIA}

Se describe el método de trabajo utilizado para el diseño y aplicación del instrumento de autoevaluación en las Instituciones de Educación Superior. Se presenta el instrumento de autoevaluación, se definen la población y la muestra y se explica el procedimiento usado para la aplicación del instrumento.

\section{Instrumento}

Para desarrollar la propuesta del instrumento de autoevaluación del desempeño organizacional en universidades fue necesario revisar los modelos teóricos utilizados para la aplicación del CMI en estas instituciones. De esta manera se tomaron en cuenta las adaptaciones de Philbin (2011), Hladchenko (2015) y Mohamad (2016). De acuerdo con esta revisión se obtuvieron los conceptos clave que aseguraran la validez de contenido del instrumento y con base en estos, se establecieron las perspectivas del cuadro de mando integral y los reactivos para cada una de ellas. Como ejercicio de validez de experto, los reactivos diseñados fueron aplicados a los directivos de una institución de educación superior tecnológica en el estado de Veracruz con el objetivo de obtener retroalimentación y realizar los ajustes necesarios al cuestionario.

El instrumento final consistió de 50 reactivos distribuidos de la siguiente manera: 10 ítems en la perspectiva financiera, los cuales evalúan la viabilidad económica y la eficiencia en el manejo de los recursos; la perspectiva clientes mide la satisfacción de estudiantes, egresados, padres de familia, sector productivo y autoridades gubernamentales mediante 10 reactivos; los procesos internos considerados fueron la docencia, investigación, vinculación, extensión de servicios y gestión administrativa, los cuales se evalúan con 20 reactivos; finalmente, se diseñaron 10 preguntas para la perspectiva aprendizaje y crecimiento las cuales consideran la capacitación y satisfacción del personal, así como el software y plataformas tecnológicas disponibles en la institución. La escala de respuestas fue tipo Likert de seis puntos: 1) Fuertemente en desacuerdo, 2) En desacuerdo, 3) Ligeramente en desacuerdo, 4) Ligeramente de acuerdo, 5) De acuerdo y 6) Fuertemente de acuerdo. 


\section{Población y muestra}

La Secretaría de Educación Pública (2016) clasifica al sistema público de educación superior mexicano en diez subsistemas, los cuales reciben diferentes tipos de financiamiento del gobierno federal, los gobiernos estatales 0 ambos. Existen en total 888 instituciones públicas de educación superior, las cuales se distribuyen de la forma que lo muestra la Tabla 1:

Tabla 1: Instituciones públicas de educación superior. Datos de SEP (2016)

\begin{tabular}{|l|c|}
\hline \multicolumn{1}{|c|}{ Subsistema } & Instituciones \\
\hline Universidades Públicas Federales & 9 \\
\hline Universidades Públicas Estatales & 34 \\
\hline Universidades Públicas Estatales con apoyo solidario & 23 \\
\hline Institutos Tecnológicos & 258 \\
\hline Universidades Tecnológicas & 104 \\
\hline Universidades Politécnicas & 51 \\
\hline Universidades Interculturales & 12 \\
\hline Centros Públicos de Investigación & 51 \\
\hline Normales Públicas & 260 \\
\hline Otras instituciones públicas & 86 \\
\hline Total & 888 \\
\hline
\end{tabular}

Para la aplicación del instrumento fueron enviados correos electrónicos a las autoridades directivas de cada institución, considerando al director o su equivalente, subdirectores y jefes de departamentos académicos. Esto con la intención de que las personas que respondieran el cuestionario tuvieran una visión global de todos los procesos de la institución.

\section{Procedimiento}

La aplicación del instrumento fue realizada por medio de la plataforma virtual Online Encuesta (https://www.onlineencuesta.com/), en la cual fue capturado el cuestionario y enviado por correo electrónico a las autoridades directivas de las universidades encuestadas. Junto con el cuestionario fueron enviadas una carta explicativa del proyecto a desarrollar, así como un acuerdo de confidencialidad y aceptación de los términos de participación. Para la recopilación de la base de datos de los correos fueron consultadas las páginas electrónicas oficiales de las instituciones, considerando los directorios públicos que en ellas aparecen.

Para el análisis de datos se utilizó el software estadístico Stata en su versión 14. Se calculó la confiabilidad del instrumento mediante el coeficiente de Alfa de Cronbach por cada una de las dimensiones del instrumento. Además, se realizó un índice por dimensión, basado en la media aritmética de los reactivos incluidos en cada una de ellas. Finalmente, se calcularon las correlaciones bivariadas de cada una de las perspectivas.

\section{RESULTADOS}

En la presente sección se muestran los resultados de la aplicación realizada: la confiabilidad del instrumento, estadísticos descriptivos por dimensión y la matriz de correlaciones. Se obtuvieron respuesta de 74 instituciones públicas de educación superior las cuales pertenecen a siete diferentes subsistemas de los 10 existentes. Respecto a la participación por estados, se tuvo repuesta de 26 entidades federativas diferentes de las 32 que existen en el país. La Tabla 2 presenta la información de las universidades de las cuales se recibió respuesta y el subsistema al cual pertenecen.

Tabla 2. Instituciones encuestadas

\begin{tabular}{|l|c|}
\hline \multicolumn{1}{|c|}{ Subsistema } & Respuestas \\
\hline Institutos Tecnológicos Superiores & 20 \\
\hline Universidades Estatales & 20 \\
\hline Universidades Tecnológicas & 15 \\
\hline Institutos Tecnológicos Federales & 9 \\
\hline Universidades Politécnicas & 7 \\
\hline Universidades Interculturales & 2 \\
\hline Universidades Federales & 1 \\
\hline Total & 74 \\
\hline
\end{tabular}




\section{Confiabilidad}

Para evaluar la confiabilidad del instrumento se utilizó el coeficiente de Alfa de Cronbach, el cual permite medir el nivel de confiabilidad de una escala a partir de las $\mathrm{n}$ variables observadas; la cual, cuanto más se aproxime a su valor máximo, 1, mayor es la confiabilidad del instrumento. Además, en determinados contextos, se considera que valores del alfa superiores a 0.7 son suficientes para garantizar la fiabilidad de la escala (Nunnally, 1988). De acuerdo con los resultados que la Tabla 3 muestra puede apreciarse que las cuatro dimensiones del instrumento tienen un coeficiente alfa mayor a 0.8 , lo cual indica que la confiabilidad es alta para cada una de ellas.

Tabla 3: Confiabilidad del instrumento

\begin{tabular}{|l|c|c|}
\hline \multicolumn{1}{|c|}{ Perspectiva } & Número de reactivos & Alfa de Cronbach \\
\hline Financiera & 10 & 0,914 \\
\hline Clientes & 10 & 0,918 \\
\hline Procesos internos & 20 & 0,941 \\
\hline Aprendizaje y crecimiento & 10 & 0,889 \\
\hline
\end{tabular}

El hecho de que se haya obtenido niveles satisfactorios de confiabilidad es indicativo de la consistencia interna de cada una de las dimensiones del instrumento, por lo que se abre la posibilidad de replicar la aplicación en otras instituciones de educación superior.

\section{Resultados descriptivos}

Respecto a la percepción por dimensión, se calculó la media aritmética de los reactivos que se incluyen en cada una de ellas. Para analizar la dispersión de los datos se presenta además la desviación estándar. La Tabla 4 resume los resultados. Puede apreciarse en los resultados obtenidos que la percepción respecto al desempeño en las perspectivas está dividida en pares. Existen dos perspectivas con mayor valoración: aprendizaje y crecimiento $(4,591)$ y clientes $(4,578)$; mientras que las perspectivas financiera y la perspectiva procesos internos tienen puntuaciones menores y muy cercanas $(4,266$ y 4,296 respectivamente).

Tabla 4: Resultados descriptivos por perspectiva

\begin{tabular}{|l|c|c|c|c|}
\hline \multicolumn{1}{|c|}{ Perspectiva } & Media & Desv. Est. & Mínimo & Máximo \\
\hline Financiera & 4,266 & 0,982 & 1,5 & 6 \\
\hline Clientes & 4,578 & 0,764 & 2,2 & 6 \\
\hline Procesos internos & 4,296 & 0,844 & 2,2 & 6 \\
\hline Aprendizaje y crecimiento & 4,591 & 0,810 & 2,5 & 6 \\
\hline
\end{tabular}

La perspectiva mejor valorada es la de aprendizaje y crecimiento que evalúa tanto el conocimiento que tienen los miembros de la organización, así como las plataformas de apoyo tecnológico de las cuales disponen. De esta forma se aprecia que los encuestados califican adecuadamente el nivel educativo con el que cuentan los integrantes de su universidad, así como su satisfacción y los procesos de capacitación brindados. Sin embargo, en esta dimensión los respondientes perciben una deficiencia en cuanto a la integración del personal como equipo de trabajo. En cuanto a la tecnología, se tiene una percepción de igual manera deficiente.

En el caso de la perspectiva clientes, se consulta acerca de la satisfacción de los interesados externos de la universidad con su desempeño. En este caso, puede apreciarse una alta percepción sobre el hecho de que las instituciones públicas son la primera opción en la elección de los estudiantes; además, acerca de la satisfacción de las autoridades públicas en la actuación de las universidades, la satisfacción de los egresados con la formación profesional recibida y de los estudiantes con el desarrollo de sus materias. De esta forma, se puede concluir que los encuestados perciben una alta satisfacción de sus clientes principales, es decir, los alumnos; no obstante, los encuestados perciben que, a pesar de ser la primera opción de los estudiantes, no son los mejores alumnos los que eligen a su institución. Se observa que esta perspectiva es la que menor desviación estándar presenta, lo que es indicativo de que en esta perspectiva existe un mayor consenso respecto a los resultados obtenidos por las distintas instituciones. 
y la financiera. Los procesos internos considerados en el cuestionario fueron las actividades sustantivas de las instituciones de educación superior: docencia, investigación, vinculación, extensión de servicios y gestión administrativa. De esta manera, en comparación con las perspectivas anteriores, esta dimensión obtuvo una percepción menor respecto del desempeño en cuanto a los resultados de aprendizaje y la calidad de la docencia, así como en la productividad científica, actividades de vinculación con el medio empresarial y tiempos de respuesta en los procesos administrativos. Puede apreciarse que en general los encuestados consideran como insuficiente la cantidad y calidad de la investigación realizada en sus instituciones, así como un proceso de docencia también débil.

Finalmente, la perspectiva financiera obtuvo la menor evaluación por parte de los encuestados. En esta, se mide la percepción respecto de la viabilidad económica de la institución y la forma en la cual se obtienen y aplican los recursos monetarios en ella. Además, acerca de la autosuficiencia en los recursos y la responsabilidad fiscal con la que son ejercidos. En esta dimensión se presenta la mayor variabilidad en las respuestas (desviación estándar de 0.982), lo que indica que existe una gran dispersión respecto a cómo los encuestados valoran el desempeño financiero de sus instituciones. El hecho de ser la dimensión calificada con el menor grado de desempeño es congruente con la naturaleza de las instituciones que fueron encuestadas, ya que al ser de carácter público, su financiamiento se encuentra garantizado por el Estado y la sustentabilidad económica y búsqueda de ingresos no son temas que representen la mayor preocupación para sus miembros.

\section{Correlaciones}

Para poder verificar el grado en el cual las perspectivas del desempeño organizacional se relacionan se calculó el coeficiente de correlación de Pearson, el cual es presentado en la Tabla 5.

Tabla 5: Matriz de correlaciones por perspectiva. * $p<0,05$

\begin{tabular}{|l|c|c|c|c|}
\hline \multicolumn{1}{|c|}{ Perspectivas } & Financiera & Clientes & $\begin{array}{c}\text { Procesos } \\
\text { internos }\end{array}$ & $\begin{array}{c}\text { Aprendizaje } y \\
\text { crecimiento }\end{array}$ \\
\hline Financiera & 1,000 & & & \\
\hline Clientes & $0,703^{*}$ & 1,000 & & \\
\hline Procesos internos & $0,730^{*}$ & $0,833^{*}$ & 1,000 & \\
\hline Aprendizaje y crecimiento & $0,630^{*}$ & $0,736^{*}$ & $0,742^{*}$ & 1,000 \\
\hline
\end{tabular}

Puede apreciarse que todos los coeficientes de correlación son estadísticamente significativos. El valor más alto lo presenta la correlación entre las perspectivas clientes y procesos internos. Esto es indicativo de la alta relación que existe entre la correcta operación de las actividades sustantivas de la universidad con la satisfacción de sus interesados, por ejemplo, la calidad de la docencia con la satisfacción de estudiantes y padres de familia o las actividades de vinculación y extensión con la satisfacción de la sociedad en general y las autoridades gubernamentales. La relación que presenta una menor intensidad se presenta entre las perspectivas financiera y la de aprendizaje y crecimiento. Esto coincide con el modelo original de Kaplan y Norton (1992), en el cual las perspectivas se relacionan en una serie de cadenas causa-efecto. La lógica del modelo es que los activos intangibles (conocimiento) de la perspectiva de aprendizaje y crecimiento mejoran los procesos internos en la organización, los cuales a su vez influyen en la satisfacción de los clientes que redundará en mejores resultados financieros. Al ser la cadena más larga la que vincula al aprendizaje y crecimiento con la perspectiva financiera, se puede considerar lógico el hecho de que la correlación entre estas dimensiones sea la de menor intensidad. Un punto a resaltar es que debido a la naturaleza de las instituciones públicas de educación superior no es adecuado considerar a la perspectiva financiera como el fin último de las mismas. Por esto se puede apreciar que su correlación con la perspectiva clientes, aunque significativa, no se encuentra entre las más altas de la matriz, ya que no necesariamente la satisfacción de los estudiantes, padres de familia y sociedad está vinculada directamente con los resultados financieros de la universidad.

\section{CONCLUSIONES}

En el presente documento se han reportado los resultados del diseño y aplicación de la prueba piloto de un instrumento de autoevaluación del desempeño organizacional para las instituciones públicas de educación superior en México. Las principales conclusiones del trabajo son: a pesar de la importancia que la autoevaluación del desempeño organizacional tiene para las universidades, no se detectó algún instrumento adecuado para su aplicación en México, por lo que se estimó adecuado el diseño de un cuestionario para tal efecto; el modelo de cuadro de mando integral ha sido utilizado y validado para medir el desempeño organizacional en las instituciones de educación superior, por lo que resultó útil como marco para establecer 
las dimensiones bajo las cuales se estructuró el instrumento; de acuerdo con los resultados obtenidos de la aplicación realizada, todas las dimensiones del cuestionario diseñado cuentan con índices de confiabilidad aceptables, por lo cual el instrumento resulta una aportación a la literatura que aborda la medición del desempeño organizacional en las instituciones de educación superior ya que considera al concepto desde diversas dimensiones y se evalúa el impacto de la organización en todas sus partes interesadas.

Las respuestas obtenidas evidencian que las universidades tienen mejor desempeño en dos perspectivas (clientes y de aprendizaje y crecimiento) sobre las dos restantes (financiera y de procesos internos). El hecho de que la dimensión financiera sea la que obtuvo una menor puntuación es congruente con la naturaleza de las instituciones de educación superior públicas; sin embargo, es importante que estas organizaciones mejoren estos indicadores ya que de esta forma garantizan su sostenibilidad a lo largo del tiempo. Por su parte, en la perspectiva de procesos internos se obtuvieron bajos puntajes en la investigación y docencia, lo que también es indicativo de la necesidad de implementar mecanismos que eleven la calidad en estas actividades fundamentales en las instituciones.

Como agenda futura de investigación queda pendiente la aplicación a una muestra de instituciones lo cual permita estudiar características psicométricas del instrumento tal como la validez de constructo mediante un análisis factorial. Finalmente, de acuerdo con los resultados de la investigación se puede recomendar a los gestores en las universidades el realizar un diagnóstico de autoevaluación del desempeño organizacional en cada institución de educación superior. Para ello puede aplicarse el instrumento diseñado a una muestra representativa de integrantes de cada institución, lo que brindaría un panorama de la percepción de los directivos y académicos acerca de lo bien que se están logrando los objetivos. Además, este diagnóstico serviría para establecer acciones que ayuden a mejorar el desempeño en las dimensiones en las que se obtengan puntuaciones bajas y de esta manera mejorar el desempeño de las instituciones de educación superior.

\section{REFERENCIAS}

Aghion, P., Dewatripont, M., Hoxby, C., Mas-Colell, A., y Sapir, A. The governance and performance of universities: evidence from Europe and the US, doi: 10.1111/j.1468-0327.2009.00238.x, Economic Policy, 25(61), 7-59 (2010)

Baboulet, O., y Lenzen, M. Evaluating the environmental performance of a university, doi: 10.1016/j.jclepro.2010.04.006, Journal of Cleaner Production, 18(12), 1134-1141 (2010)

Barra, A. M., y Gómez, N. M. Análisis del rendimiento organizacional a través del modelo gratton. caso aplicado a una carrera de pregrado de una universidad estatal chilena, doi: 10.4067/S071850062013000400005, Formación Universitaria, 6(4), 39-48 (2013)

Capaldi, E. D., y Abbey, C. W. Performance and Costs in Higher Education: A Proposal for Better Data, doi: 10.1080/00091383.2011.550250 Change, 43(2), 8-15 (2011)

Capaldi, E. D., Lombardi, J. V, Abbey, C. W., y Craig, D. D. The Top American Research Universities. Generic, The Center for Measuring University Performance: Arizona, E.E.U.U (2010)

Guthrie, J., y Neumann, R. Economic and non-financial performance indicators in universities, doi: 10.1080/14719030701340390 Public Management Review, 9(2), 231-252 (2007)

Hladchenko, M. Balanced Scorecard - a strategic management system of the higher education institution, doi: 10.1108/IJEM-11-2013-0164, International Journal of Educational Management, 29(2), 167-176 (2015)

Iqbal, M. J., Rasli, A., Low Hock, H., Huam Hon, T., Mohamad Bin Bilal, A., y Hassan, I. Knowledge Economy and University Performance, doi: 10.7813/2075-4124.2011/3-5/A.5, International Journal of Academic Research, 3(5), 27-32 (2011)

Jarrat, A. Report of the Steering Committee for Efficiency Studies in Universities. Book, Committee of ViceChancellors and Principals of the United Kingdom Universities: Londres (1985)

Kaplan, R. S., y Norton, D. P. The Balanced Scorecard: Measures that Drive Performance. Harvard Business Review, (Ene-Feb), 71-79 (1992)

Mertens, P. Comparative Indicators for German Universities, https://eric.ed.gov/?id=EJ203399, ISSN: 09543716, International Journal of Institutional Management in Higher Education, 3(1), 155-168 (1979) 
Mohamad, B. A. M. The Assessment of Accredited Faculties Performance at Assiut University by Using Balanced Scorecard (BSC), doi: 10.19044/esj.2016.v12n22p249, European Scientific Journal, 12(22) (2016)

Mustafa, S. T., y Dalen, C. Dimensions of Quality in Higher Education: How Academic Performance Affects University Students' Teacher Evaluations, ISSN: ISSN: 1540 - 7780, Journal of American Academy of Business, Cambridge, 8(1), 294-303 (2006)

Nunnally, J. C. Psycometric Theory. Book, McGraw Hill: New Jersey (1988)

Palomares-Montero, D., y García-Aracil, A. What are the key indicators for evaluating the activities of universities?, doi: 10.3152/095820211X13176484436096, Research Evaluation, 20(5), 353-363 (2011)

Philbin, S. P. Design and implementation of the Balanced Scorecard at a university institute, doi: 10.1108/13683041111161148, Measuring Business Excellence, 15(3), 34-45 (2011)

Pounder, J. Organizational Effectiveness in Higher Education, doi: 10.1177/0263211X990274006 Educational Management \& Administration, 27(4), 389-400 (1999)

Tlapa, D. A., Limón, J., y Báez, Y. A. Gestión de la Calidad y del Medio Ambiente en Instituciones de Educación Superior mediante Integración de ISO 9001 e ISO 14001, doi: 10.4067/S0718-50062009000200006, Formación Universitaria, 2(2), 35-46(2009)

Sarrico, C., Rosa, M., Teixeira, P., y Cardoso, M. Assessing Quality and Evaluating Performance in Higher Education: Worlds Apart or Complementary Views?, doi: 10.1007/s11024-010-9142-2, Minerva: A Review of Science, Learning \& Policy, 48(1), 35-54 (2010)

Secretaría de Educación Publica, (SEP). Instituciones de Educación Superior, https://goo.gl/VMxvJ3, acceso 3 de agosto de 2016, México (2016)

Shin, J. Classifying higher education institutions in Korea: a performance-based approach. Higher Education, doi: 10.1007/s10734-008-9150-4, 57(2), 247-266 (2009)

Shun-Hsing, C., Ching-Chow, Y., y Jiun-Yan, S. The application of balanced scorecard in the performance evaluation of higher education, doi: 10.1108/09544780610647892 The TQM Magazine, 18(2), 190-205 (2006)

Tarí, J. J., y De Juana Espinosa, S. EFQM model self-assessment using a questionnaire approach in university administrative services, doi: 10.1108/09544780710828449, The TQM Magazine, 19(6), 604-616 (2007)

Wu, H.-Y., Chen, J.-K., Chen, I. S., y Zhuo, H.-H. Ranking universities based on performance evaluation by a hybrid MCDM model, doi: 10.1016/j.measurement.2012.02, Measurement, 45(5), 856-880 (2012)

Xing, Z. Impact of University's Optimal Human Resource Management Practices on Organizational Performance, doi: 10.1016/s1874-8651(10)60084-1, Systems Engineering - Theory \& Practice, 29(11), 112$122(2009)$ 